\title{
Moist exposed burn ointment promotes cutaneous excisional wound healing in rats involving VEGF and bFGF
}

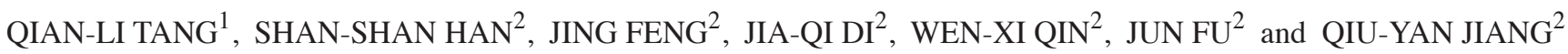 \\ ${ }^{1}$ Scientific Experimental Center, Youjiang Medical University for Nationalities, Baise, Guangxi 533000; \\ ${ }^{2}$ The First Affiliated Hospital, Guangxi University of Chinese Medicine, Nanning, Guangxi 530023, P.R. China
}

Received May 28, 2013; Accepted January 16, 2014

DOI: $10.3892 / \mathrm{mmr} .2014 .1921$

\begin{abstract}
Cutaneous delayed wounds are a challenging clinical problem, and vascular endothelial growth factor (VEGF) and basic fibroblast growth factor (bFGF) exhibit key roles in wound healing. Moist exposed burn ointment (MEBO), a Chinese burn ointment with a USA patented formulation, has been reported to promote chronic ischemic and neurogenic ulcer healing in patients; however, the underlying mechanisms remain unclear. In the present study, MEBO significantly promoted the formation of granulation tissue in cutaneous excisional wounds, shortened the time of wound healing, and increased neovascularization and the number of fibroblasts. Furthermore, as well as enhancing the protein expression, MEBO application also increased the gene expression of VEGF and bFGF. The results indicate that MEBO promotes cutaneous excisional wound healing by at least partially enhancing VEGF and bFGF production, implicating the potential uses of MEBO for delayed cutaneous wound healing.
\end{abstract}

\section{Introduction}

Cutaneous wounds, known as ulcers, are an extremely common clinical problem and often arise following acute or chronic mechanical causes, physical or chemical burns, frostbite, infections, and disorders, including rheumatism, diabetes, peripheral vascular disease, lipodermatosclerosis and malignant tumors $(1,2)$. Furthermore, cutaneous wound healing may be significantly delayed due to the aforementioned factors. At present, delayed cutaneous wounds are one of major burdens for health care, and lead to a reduced quality of life in patients who suffer from this type of wound $(3,4)$.

Correspondence to: Professor Qiu-Yan Jiang, The First Affiliated Hospital, Guangxi University of Chinese Medicine, 89-9 Dongge Road, Nanning, Guangxi 530023, P.R. China

E-mail: qiuyanjiang2011@163.com

Key words: traditional Chinese medicine, moist exposed burn ointment, wound healing, growth factors
Wound healing is a complicated biological process involving a series of dynamic events, including hemostasia, inflammation, cell proliferation and differentiation, neovascularization, granulation tissue formation, collagen synthesis, epithelialization, and wound contraction. Increasing evidence has established the hypothesis that growth factors, including vascular endothelial growth factor (VEGF) and basic fibroblast growth factor (bFGF), are key regulators of normal and abnormal angiogenesis, and tissue repair in animals and humans (5-8). VEGF promotes angiogenesis/vasculogenesis and vascular permeability, and enhances endothelial cell proliferation and migration as well as the adhesion of leukocytes $(5,9)$. Further research revealed that VEGF stimulates hydrogen sulfide synthesis and release from endothelial cells, thus leading to subsequent endothelial cell growth, migration and permeability, microvessel formation, and wound healing (10). In addition, VEGF promotes epithelialization and collagen deposition in the wound (11). FGF-2, known as bFGF, is a member of a large FGF family and induces angiogenesis, endothelial cell and fibroblast proliferation, and wound healing (12-14). Recent data indicated that bFGF-mediated angiogenesis refers to endothelial cell proliferation, migration and tube formation by activating c-Jun $\mathrm{N}$-terminal kinase/stress-activated protein kinase signaling (15). Notably, the expression of VEGF and bFGF was increased following skin injury, particularly in the early stages of healing, showing greatest intensity at the center of the wound, with progressive decline in intensity towards the periphery and almost no VEGF or bFGF in uninjured skin $(16,17)$. VEGF and bFGF, however, are decreased in delayed cutaneous wounds, including diabetic wounds and chronic ulcers (17-19). Inhibition of VEGF and bFGF by neutralizing antibodies results in a decrease in the migration of fibroblasts and a delay in wound healing (20), while treatment with recombinant bFGF and VEGF, or overexpression of VEGF accelerates wound healing $(14,21,22)$. Therefore, it is a potentially clinically beneficial to increase the levels of VEGF and bFGF in cutaneous wounds, particularly in delayed wound healing.

In the past few decades, moist exposed burn ointment (MEBO), a Chinese burn ointment with a USA patented formulation since 1995, which was developed at the China National Science and Technology Centre in Beijing in 1989 (23,24), has been used to treat thickness burns in clinical practice and has achieved beneficial efficacy (25-28). MEBO contains 
sesame oil, $\beta$-sitosterol, berberine, and other small quantities of plant ingredients from Chinese herbal remedies, including Coptis chinensis Franch., Scutellaria baicalensis Georgi, Phellodendron Chinese Schneid., Pheretima aspergillum (E Perrier) and Papaver somniferum L. (29). Further research indicated that $\beta$-sitosterol exhibits anti-inflammatory effects (30) and that berberine exhibits antimicrobial effects (31). Clinical and experimental studies have shown that MEBO exhibits analgesic and antimicrobial effects, and reduces the treatment time of burns $(25-28,32,33)$. Furthermore, MEBO induces debridement and epithelial repair, associated with improved scar quality and reduced costs of treatment for patients with burns $(25,27,28,33)$. MEBO has been observed to promote chronic ischemic and neurogenic ulcer healing, and reduces the time of wound healing in patients $(34,35)$; however, the underlying mechanisms remain unclear.

The aim of the present study was to investigate the effects of MEBO on cutaneous excisional wounds in experimental rats and to explore the underlying mechanisms.

\section{Materials and methods}

Reagents. MEBO was purchased from Shantou MEBO Pharmaceuticals Co., Ltd. (Shantou, China). Recombinant bovine bFGF (rb-bFGF) was purchased from Zhuhai Yisheng Biological Pharmaceutical Co. (Zhuhai, China). Rabbit polyclonal antibodies against VEGF and bFGF were purchased from Abcam (Cambridge, MA, USA), and secondary mouse anti-rabbit peroxidase-conjugated monoclonal antibody was obtained from Sigma-Aldrich (St. Louis, MO, USA). TRIzol reagent was purchased from Invitrogen Life Technologies (Carlsbad, CA, USA). A bicinchoninic acid (BCA) protein assay kit was purchased from Thermo Fisher Scientific (Waltham, MA, USA).

Wound preparation and experimental design. Sixty male Sprague-Dawley rats (age, 8-weeks; weight, 220-250 g) were purchased from the Animal Experimental Center of Guangxi Medical University (Nanning, China; SCXKGui2009-0002). The study was approved by the Ethics Committees of Youjiang Medical University for Nationalities (Baise, China) and Guangxi University of Chinese Medicine. Rats were housed in a temperature- and humidity-controlled room with a 12-h ligh/dark cycle and had free access to food and water. Following 1-week acclimatization, cutaneous full-thickness excisional wounds were prepared as previously described with some modifications (36). Briefly, the rat hair was shaved on the dorsal side following anesthesia with pentobarbital sodium (39 $\mathrm{mg} / \mathrm{kg}$ ), and the skin was cleaned with $70 \%$ ethanol. A 2.4-cm diameter full-thickness skin defect was created on the back by skin punch biopsy under aseptic conditions. Two wounds were created in each rat. Next, the rats were randomly divided into three groups based on different treatments: Model $(n=20), \operatorname{MEBO}(n=20)$ and rb-bFGF $(n=20)$. The wounds in the model group were covered with a single dressing soaked with physiological saline and double dry sterile dressings; wounds in the MEBO group were covered with dry sterile dressings following direct coating of the wound with $1 \mathrm{~mm}$ thick MEBO; and wounds in the rb-bFGF group were covered with dry sterile dressings following by spraying of the wound with
rb-bFGF. The sterile dressings were changed daily following clearing of wound liquefaction with sterile dressings. All procedures were in accordance with internationally accepted principles for laboratory animal use and care, as found in the European Community Guidelines (EEC Directive of 1986; $86 / 609 /$ EEC) and the US guidelines (NIH publication \#85-23, revised in 1985).

Histopathological observation. Rats were euthanized using pentobarbital sodium. Granulation tissue was collected from eight-day-old wounds not containing healthy skin margin. Each tissue was fixed using $10 \%$ formalin, processed for paraffin embedding and stored at $4^{\circ} \mathrm{C}$. The following parameters were evaluated with hematoxylin and eosin (H\&E) staining on multiple serial sections $(4-5 \mu \mathrm{m})$ : Neovascularization, fibroblast proliferation and inflammatory cell infiltration. All analysis was performed at x200 magnification.

Western blot analysis. The protein expression of VEGF and bFGF in granulation tissue from eight-day-old wounds was determined by western blotting, as previously described with certain modifications (37). Briefly, tissue homogenates from wound granulation were prepared using RIPA lysis buffer. Insoluble material was removed by centrifugation $\left(12,000 \mathrm{x} \mathrm{g}\right.$ for $20 \mathrm{~min} ; 4^{\circ} \mathrm{C}$; Thermo Forma, Osteroden, Germany), and the protein concentrations of the supernatants were determined using a BCA protein assay kit. Equal quantities of protein $(50 \mu \mathrm{g})$ were separated via SDS-PAGE. The protein was electrophoretically transferred to polyvinylidene difluoride membranes (Millipore, Billerica, MA, USA). The blots were incubated with the primary antibody overnight at $4^{\circ} \mathrm{C}$ and then with the secondary antibody for $1-2 \mathrm{~h}$ at room temperature. The protein bands were detected by an Enhanced Cheminluminescent Plus detection reagent kit (Amersham Pharmacia Biotech, Amersham, UK).

Quantification of $m R N A$ levels by reverse transcription-polymerase chain reaction $(R T-P C R)$. Total RNA was extracted from eight-day-old wound granulation tissue using TRIzol reagent according to the manufacturer's instructions, and subsequently transcribed into cDNA. cDNA was then transcribed into mRNA by RT-PCR. The product was separated via agarose gel electrophoresis. The sequences of primers used were as follows: Forward: 5'-CGGAAGATTAGGGAGTTTTG-3' and reverse: 5'-AGGGATGGGTTTGTCGTGT-3' for VEGF; forward: 5'-GCGTCCGGGAGAAGAGCGAC-3' and reverse: 5'-GCCAGGTACCGGTTCGCACA-3' for bFGF; forward: 5'-CAGTGCCAGCCTCGTCTCAT-3' and reverse: 5'-AGGGGCCATCCACAGTCTTC-3' for GAPDH; and forward: 5'-CACCCGCGAGTACAACCTTC-3' and reverse: 5'-CCCATACCCACCATCACACC-3' for $\beta$-actin.

Statistical analysis. The data are presented as the mean \pm standard deviation. Statistical analysis was performed using the SPSS 17.0 for Windows (SPSS Inc., Chicago, IL, USA). Significant differences among groups were analyzed by one-way analysis of variance, and differences between means were determined by Fisher's least significant difference post hoc test. $\mathrm{P}<0.05$ was considered to indicate a statistically significant difference. 


\section{Results}

$M E B O$ promotes the formation of granulation tissue and wound healing. MEBO was initially successfully designed to treat burns. Previously, MEBO was observed to promote chronic ulcer healing $(34,35)$; however, the underlying mechanisms remain to be fully elucidated. In the current study, a full-thickness skin defect was induced by skin punch biopsy, and treatment with MEBO for eight days significantly promoted the formation of granulation tissue when compared with the model group. Furthermore, the time of wound healing in the MEBO group was shorter compared with that of the model group $(\mathrm{P}<0.01$; Fig. 1$)$. In addition, rb-bFGF was designed as a positive control medicine for MEBO. Consistent with MEBO, rb-bFGF also caused the formation of granulation tissue and shortened the time of wound healing when compared with the model group $(\mathrm{P}<0.01)$; however, the time of wound healing in the MEBO group was also shorter than that of the rb-bFGF group $(\mathrm{P}<0.05)$. The data indicate that MEBO promotes cutaneous excisional wound healing, similar to that of rb-bFGF, indicating that there is a similarity in the effects of MEBO and rb-bFGF in the treatment of wounds.

MEBO increases neovascularization and fibroblasts in granulation tissue. To determine the mechanisms underlying the effects of MEBO, the histology of granulation tissue in three groups was observed following wound treatment for eight days. As shown in Fig. 2, there were numerous novel capillaries and fibroblasts in granulation tissue in the MEBO group, which were more abundant compared with the model group and rb-bFGF group, when observed at x200 magnification. In addition, rb-bFGF treatment for eight days resulted in marked increases in the numbers of capillaries and fibroblasts in granulation tissue when compared with the model group. These data suggest that MEBO promotes neovascularization and enhances fibroblast proliferation and/or migration.

Effects of MEBO on the protein expression of VEGF and $b F G F$. MEBO promoted neovascularization and increased fibroblasts in granulation tissue. As previously described, VEGF and bFGF levels are closely correlated with neovascularization and fibroblast proliferation (5-8). To further determine the association between MEBO and VEGF/bFGF, western blotting was used to determine the growth factor levels. Local administration of MEBO for eight days markedly increased the levels of VEGF and bFGF by $\sim 77.5$ and $90.8 \%$, respectively (all $\mathrm{P}<0.01$; Fig. 3), when compared with the model group. VEGF and bFGF protein expression in the rb-bFGF group were higher compared with the model group (all $\mathrm{P}<0.01$ ). Notably, the levels of VEGF and bFGF in the MEBO group were higher compared with the rb-bFGF group $(\mathrm{P}<0.05$ and $\mathrm{P}<0.01$, respectively). The results indicate that $\mathrm{MEBO}$ at least partially increases the protein expression levels of VEGF and bFGF to promote wound healing.

Effects of MEBO on the gene expression of VEGF and bFGF. qPCR analysis (Fig. 4) indicated that MEBO treatment for eight days led to increases in the mRNA expression of VEGF and bFGF by 40.9 and $97.1 \%$, respectively, when compared

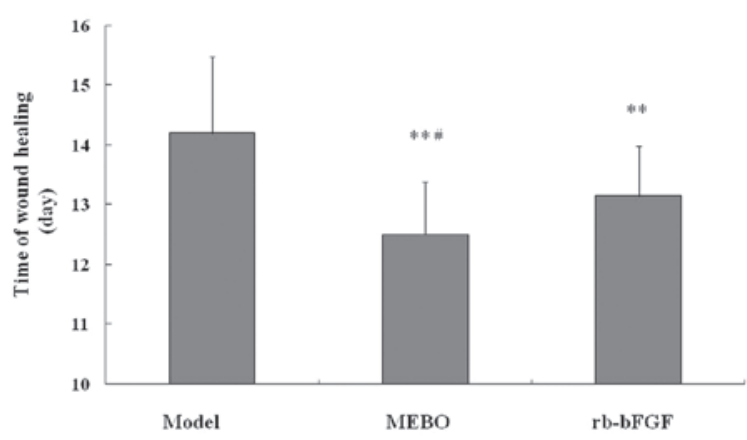

Figure 1. MEBO reduced the time of wound healing. Following treatment, the time of wound healing of the rats in each group was recorded. ${ }^{* *} \mathrm{P}<0.01$, vs. the model group; ${ }^{\text {}} \mathrm{P}<0.05$, vs. the recombinant bovine-basic fibroblast growth factor (rb-bFGF) group, $n=20$. MEBO, moist exposed burn ointment.
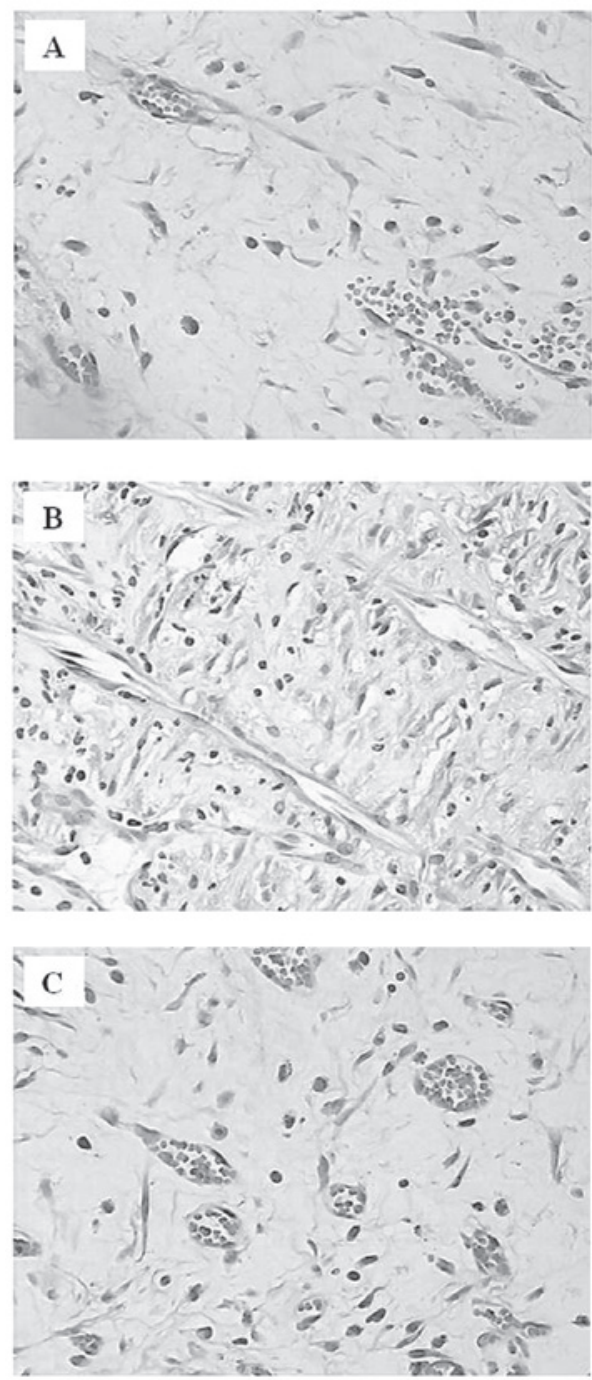

Figure 2. Effects of MEBO on the histopathology of the wounds. Granulation tissue was collected from eight-day-old wounds and then analyzed histopathology using hematoxylin and eosin staining (magnification, $\mathrm{x} 200$ ). (A) Model group, (B) MEBO group and (C) recombinant bovine-basic fibroblast growth factor group. MEBO, moist exposed burn ointment.

with the model group (all $\mathrm{P}<0.01$ ). The rb-bFGF treatment also increased the gene expression of VEGF and bFGF (all $\mathrm{P}<0.01$ ). Furthermore, the mRNA levels of VEGF and bFGF genes in 
A

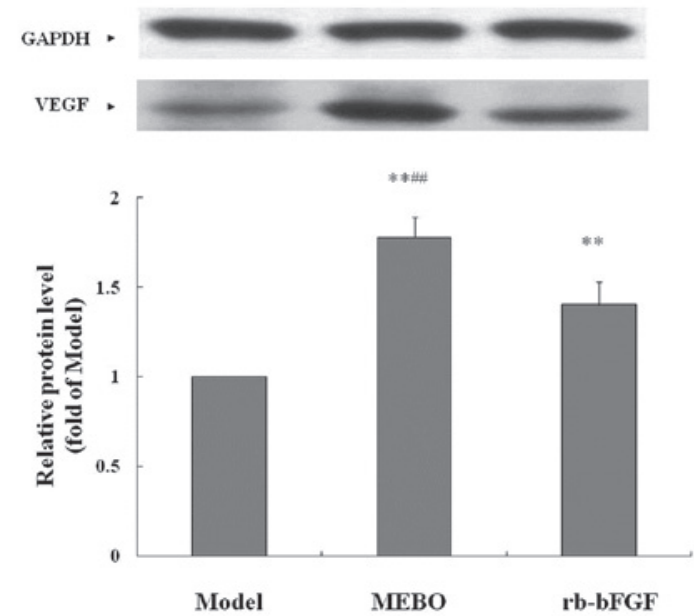

B

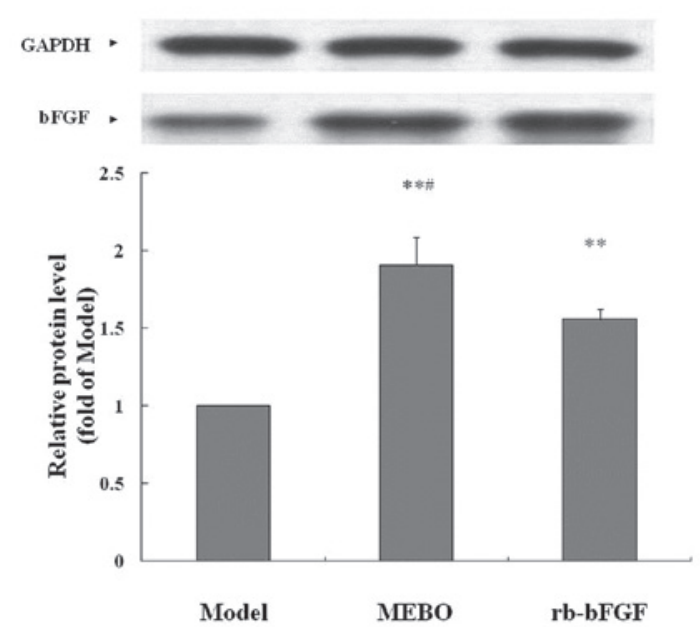

Figure 3. MEBO enhanced the protein expression of VEGF and bFGF. Following treatment for eight days, granulation tissue was collected, and the protein expression in the granulation tissue was determined by western blot analysis. (A) Relative protein level of VEGF. (B) Relative protein level of bFGF. ${ }^{* *} \mathrm{P}<0.01$, vs. the model group; ${ }^{\#} \mathrm{P}<0.05$ and ${ }^{\# \#} \mathrm{P}<0.01$, vs. the rb-bFGF group. MEBO, moist exposed burn ointment; VEGF, vascular endothelial growth factor; rb-bFBF, recombinant bovine-basic fibroblast growth factor.

the MEBO group were higher compared with the rb-bFGF group (all $\mathrm{P}<0.05$ ).

\section{Discussion}

In China and other Asian countries, traditional Chinese medicine has been widely used to treat wounds in clinical practice due to its beneficial effects; however, convincing evidence is lacking, and the mechanisms remain unclear.

In the present study, MEBO, a Chinese herbal ointment, promotes granulation tissue formation and reduces the time of wound healing in cutaneous excisional wounds, suggesting that it is effective for wound healing, which is consistent with the results of previous studies $(34,35,38)$. It is well established that excisional wounds invariably destroy tissue integrity, and lead to vascular injury and fibrin-fibronectin clot formation, thus leading to platelet recruitment, and subsequently upregulation of growth factors and cytokines, including VEGF and bFGF $(5,39)$, which triggers the forma-

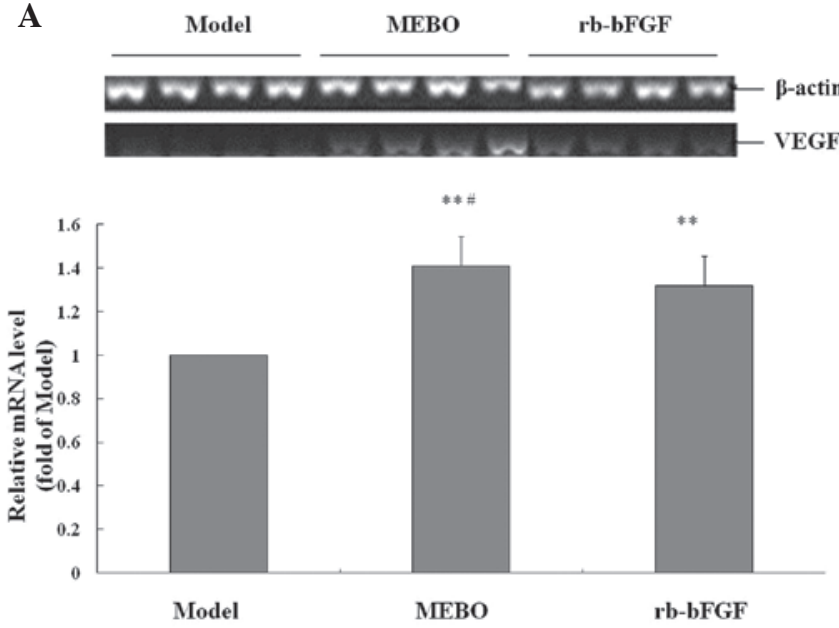

B
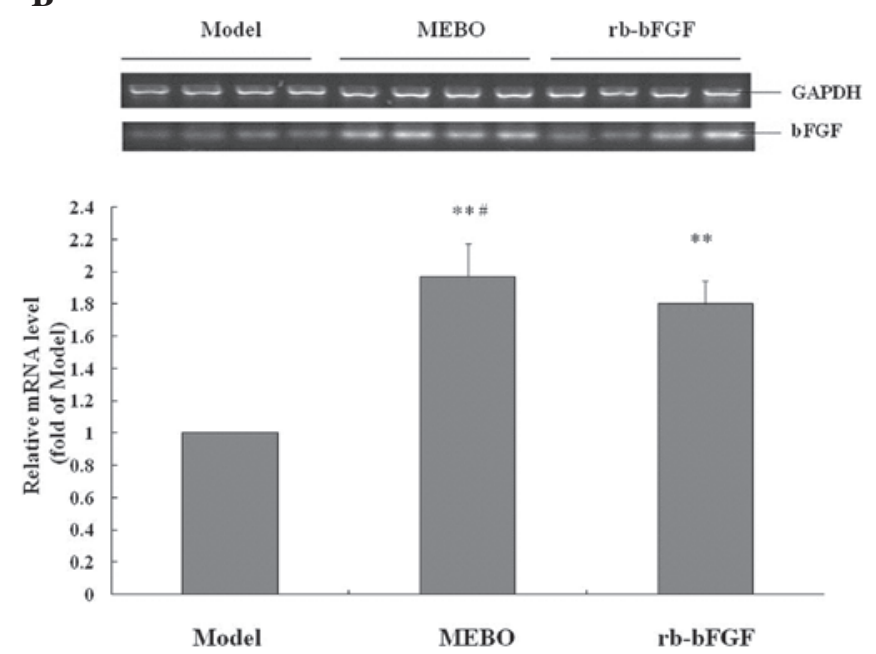

Figure 4. MEBO increased the gene expression of VEGF and bFGF. Total RNA was extracted from eight-day-old wound granulation, and mRNA level was analyzed by quantitative polymerase chain reaction. (A) Relative mRNA level of VEGF. (B) Relative mRNA level of bFGF. ${ }^{* *} \mathrm{P}<0.01$, vs. the model group; ${ }^{~} \mathrm{P}<0.05$, vs. the rb-bFGF group. MEBO, moist exposed burn ointment; VEGF, vascular endothelial growth factor; rb-bFBF, recombinant bovine-basic fibroblast growth factor.

tion of granulation tissue and wound healing. The wound healing process involves migration and proliferation of cells, including vascular endothelial cells and fibroblasts. Further investigation has demonstrated that MEBO increases neovascularization in the granulation tissue, implicating the beneficial effects of MEBO on vascular endothelial cell proliferation. Furthermore, MEBO also increased fibroblasts in the granulation tissue. Increases in the number of fibroblasts primarily arise due to the proliferation of resident fibroblasts in response to growth factors, including bFGF, and fibroblasts migrating from the surrounding connective tissue into the wound site (40). It was observed that recombinant bFGF accelerates the wound healing process $(14,41)$, thus, rb-bFGF was used as a positive control for MEBO. In the current study, rb-bFGF promoted the formation of granulation tissue, increased neovascularization and the number of fibroblasts in the granulation tissue, and reduced the time of wound healing, which were all consistent with the effects of MEBO. 
It is generally accepted that VEGF induces endothelial cell proliferation and migration, promotes vascular permeability and angiogenesis, increases collagen deposition (5,9-11), and that bFGF mediates angiogenesis, promotes the proliferation and migration of endothelial cells and fibroblasts proliferate and migration $(12-15,42)$. Therefore, it was inferred that MEBO promotion of wound healing in rats involves growth factors, including VEGF and bFGF. In order to confirm this hypothesis, western blot analysis and RT-PCR were respectively used to analyze the protein and mRNA expression of VEGF and bFGF. Notably, MEBO enhanced the protein expression of VEGF and bFGF and also elevated their mRNA expression. A previous study reported that MEBO enhanced $\alpha$-smooth muscle actin in fibroblasts, indicating that MEBO activates fibroblasts (43). It is hypothesized that leukocytes, including fibroblasts and endothelial cells are the primary origin of VEGF and bFGF (41). Furthermore, bFGF upregulates the expression of VEGF $(9,41)$. Decreased levels of VEGF and/or bFGF, or inhibition of their activity leads to the limited migration of fibroblasts and the delay of wound healing $(20,44)$. In the present study, rb-bFGF enhanced the protein and gene expression of VEGF and bFGF, which is similar to the results of a previous study (45). It is well known that the expression of VEGF and bFGF is increased following skin injury, particularly in the early stage of healing. A previous report showed that the acute wound fluid, which is rich in VEGF and bFGF, increases the mRNA levels of VEGF and bFGF (45). Since a similar effect between MEBO and rb-bFGF in treating cutaneous excisional wounds was observed, it was concluded that MEBO promotes wound healing by increasing VEGF and bFGF production.

Furthermore, compared with rb-bFGF, MEBO took less time to promote wound healing, and led to a higher production of VEGF and bFGF. In addition, increasing evidence indicates that MEBO promotes wound contraction and improves scar quality of wounds $(43,46)$. Therefore, MEBO is hypothesized to be an ideal therapy for cutaneous wounds.

In conclusion, MEBO promotes cutaneous excisional wound healing by at least partially enhancing the production of VEGF and bFGF, which reveals part of the underlying mechanism and suggests the use of MEBO for the treatment of delayed healing cutaneous wounds.

\section{Acknowledgements}

This study was supported by grants from the National Natural Science Foundation of China (grant no. 30860356) and the Guangxi Natural Science Foundation (grant no. 2013GXNSFDA019020).

\section{References}

1. Ndip A, Ebah L and Mbako A: Neuropathic diabetic foot ulcers - evidence-to-practice. Int J Gen Med 5: 129-134, 2012.

2. Herouy Y, Kreis S, Mueller T, et al: Inhibition of angiogenesis in lipodermatosclerosis: Implication for venous ulcer formation. Int J Mol Med 24: 645-651, 2009.

3. Letizia M, Uebelhor J and Paddack E: Providing palliative care to seriously ill patients with nonhealing wounds. J Wound Ostomy Continence Nurs 37: 277-282, 2010.

4. Jiang Y, Huang S, Fu X, et al: Epidemiology of chronic cutaneous wounds in China. Wound Repair Regen 19: 181-188, 2011.
5. Soyer T, Ayva S, Aliefendioğlu D, et al: Effect of phototherapy on growth factor levels in neonatal rat skin. J Pediatr Surg 46 : 2128-2131, 2011.

6. Ching YH, Sutton TL, Pierpont YN, Robson MC and Payne WG The use of growth factors and other humoral agents to accelerate and enhance burn wound healing. Eplasty 11: e41, 2011.

7. Shi L, Chang Y, Yang Y, et al: Activation of JNK signaling mediates connective tissue growth factor expression and scar formation in corneal wound healing. PLoS One 7: e32128, 2012.

8. Kajdaniuk D, Marek B, Borgiel-Marek H and Kos-Kudła B Vascular endothelial growth factor (VEGF)-part 1: in physiology and pathophysiology. Endokrynol Pol 62: 444-455, 2011.

9. Nissen NN, Polverini PJ, Koch AE, et al: Vascular endothelial growth factor mediates angiogenic activity during the proliferative phase of wound healing. Am J Pathol 152: 1445-1452, 1998.

10. Papapetropoulos A, Pyriochou A, Altaany Z, et al: Hydrogen sulfide is an endogenous stimulator of angiogenesis. Proc Natl Acad Sci USA 106: 21972-21977, 2009.

11. Bao P, Kodra A, Tomic-Canic M, et al: The role of vascular endothelial growth factor in wound healing. J Surg Res 153: 347-358, 2009.

12. Nissen NN, Polverini PJ, Gamelli RL and DiPietro LA: Basic fibroblast growth factor mediates angiogenic activity in early surgical wounds. Surgery 119: 457-465, 1996

13. Bikfalvi A, Klein S, Pintucci G and Rifkin DB: Biological roles of fibroblast growth factor-2. Endocr Rev 18: 26-45, 1997.

14. Yan L, Wu W, Wang Z, et al: Comparative study of the effects of recombinant human epidermal growth factor and basic fibroblast growth factor on corneal epithelial wound healing and neovascularization in vivo and in vitro. Ophthalmic Res 49: 150-160, 2012.

15. Kaikai S, Yuchen S, Lili J and Zhengtao W: Critical role of c-Jun $\mathrm{N}$-terminal kinase in regulating bFGF-induced angiogenesis in vitro. J Biochem 150: 189-197, 2011.

16. Gu TM, Niu XT and Guo H: Clinical observation on changes of fibroblast growth factor in burn wounds. Zhongguo Xiu Fu Chong Jian Wai Ke Za Zhi 14: 261-263, 2000 (In Chinese).

17. Lauer G, Sollberg S, Cole M, et al: Expression and proteolysis of vascular endothelial growth factor is increased in chronic wounds. J Invest Dermatol 115: 12-18, 2000.

18. Zandifar E, Sohrabi Beheshti S, Zandifar A and Haghjooy Javanmard S: The effect of captopril on impaired wound healing in experimental diabetes. Int J Endocrinol 2012: 785247, 2012.

19. Peplow PV and Baxter GD: Gene expression and release of growth factors during delayed wound healing: a review of studies in diabetic animals and possible combined laser phototherapy and growth factor treatment to enhance healing. Photomed Laser Surg 30: 617-636, 2012.

20. Lee EY,Xia Y, Kim WS, et al: Hypoxia-enhanced wound-healing function of adipose-derived stem cells: increase in stem cell proliferation and up-regulation of VEGF and bFGF. Wound Repair Regen 17: 540-547, 2009.

21. Nauta A, Seidel C, Deveza L, et al: Adipose-derived stromal cells overexpressing vascular endothelial growth factor accelerate mouse excisional wound healing. Mol Ther 21: 445-455, 2013.

22. Anghel A, Mut-Vitcu B, Savu L, et al: Clinical improvement after treatment with VEGF(165) in patients with severe chronic lower limb ischaemia. Genomic Med 1: 47-55, 2007.

23. Rongxiang X: On the Principles of Treatment of Burn Wound. Beijing: China National Science and Technology Centre for Burns, Wounds and Ulcers, 1994.

24. Atiyeh BS, Dham R, Kadry M, et al: Benefit-cost analysis of moist exposed burn ointment. Burns 28: 659-663, 2002.

25. Ang ES, Lee ST, Gan CS, et al: The role of alternative therapy in the management of partial thickness burns of the face - experience with the use of moist exposed burn ointment (MEBO) compared with silver sulphadiazine. Ann Acad Med Singapore 29: 7-10, 2000.

26. Allam AM, Mostafa W, Zayed E and El-Gamaly J: Management of the acute partial-thickness burned hand; moist exposed burn ointment or silver sulphadiazine cream both combined with a polyethylene bag. Ann Burns Fire Disasters 20: 144-148, 2007.

27. Hirsch T, Ashkar W, Schumacher O, et al: Moist exposed burn ointment (MEBO) in partial thickness burns - a randomized, comparative open mono-center study on the efficacy of dermaheal (MEBO) ointment on thermal 2nd degree burns compared to conventional therapy. Eur J Med Res 13: 505-510, 2008.

28. Carayanni VJ, Tsati EG, Spyropoulou GC, Antonopoulou FN and Ioannovich JD: Comparing oil based ointment versus standard practice for the treatment of moderate burns in Greece: a trial based cost effectiveness evaluation. BMC Complement Altern Med 11: 122, 2011. 
29. Yong YL: Analysis of MEBO cream. Institute of Science and Forensic Medicine, Department of Scientific Services, Health Science Division, Singapore 1999, Report no. 99033191.

30. Valerio $M$ and Awad AB: $\beta$-Sitosterol down-regulates some pro-inflammatory signal transduction pathways by increasing the activity of tyrosine phosphatase SHP-1 in J774A.1 murine macrophages. Int Immunopharmacol 11: 1012-1017, 2011.

31. Zou Q, Li Y, Zhang L, et al: Antibiotic delivery system using nano-hydroxyapatite/chitosan bone cement consisting of berberine. J Biomed Mater Res A 89: 1108-1117, 2009.

32. Jewo PI, Fadeyibi IO, Babalola OS, et al: A comparative study of the wound healing properties of moist exposed burn ointment (MEBO) and silver sulphadiazine. Ann Burns Fire Disasters 22: 79-82, 2009.

33. Jurjus A, Atiyeh BS, Abdallah IM, et al: Pharmacological modulation of wound healing in experimental burns. Burns 33 892-907, 2007

34. Wang QS, Tang QL, Zhang L, et al: MEBO for treating 47 cases of chronic ischemic ulcer in lower limber. Chin J Burns Wound Surface Ulcer 17: 296-297, 2005 (In Chinese).

35. Li JH, Zhang L, Tang QL, et al: Clinical observation on effect of MEBO on neurogenic ulcer. Liaoning J Tradit Chin Med 39: 1095-1096, 2012 (In Chinese)

36. Hong JW, Lee WJ, Hahn SB, Kim BJ and Lew DH: The effect of human placenta extract in a wound healing model. Ann Plast Surg 65: 96-100, 2010.

37. Feng XT, Wang TZ, Chen Y, et al: Pollen Typhae total flavone improves insulin-induced glucose uptake through the $\beta$-arrestin-2-mediated signaling in $\mathrm{C} 2 \mathrm{C} 12$ myotubes. Int J Mol Med 30: 914-922, 2012.
38. Cho KY, Lee SJ, Burm JS and Park EA: Successful combined treatment with total parenteral nutrition fluid extravasation injuries in preterm infants. J Korean Med Sci 22: 588-594, 2007.

39. Furie B and Furie BC: Molecular and cellular biology of blood coagulation. N Engl J Med 326: 800-806, 1992.

40. Desmoulière A, Chaponnier C and Gabbiani G: Tissue repair, contraction, and the myofibroblast. Wound Repair Regen 13: $7-12,2005$.

41. Lee E, Kim DY, Chung E, et al: Transplantation of cyclic stretched fibroblasts accelerates the wound-healing process in streptozotocin-induced diabetic mice. Cell Transplant. Feb 4 2013 (Epub ahead of print).

42. Kanazawa S, Fujiwara T, Matsuzaki S, et al: bFGF regulates PI3-kinase-Rac1-JNK pathway and promotes fibroblast migration in wound healing. PLoS One 5: e12228, 2010.

43. El Kahi CG, Atiyeh BS, Abdallah Hajj Hussein I, et al: Modulation of wound contracture alpha-smooth muscle actin and multispecific vitronectin receptor integrin alphavbeta 3 in the rabbit's experimental model. Int Wound J 6: 214-224, 2009.

44. Lee HJ, Kwon JY, Shin SW, et al: Effects of sevoflurane on collagen production and growth factor expression in rats with an excision wound. Acta Anaesthesiol Scand 54: 885-893, 2010.

45. Koenen P, Spanholtz TA, Maegele M, et al: Acute and chronic wound fluids inversely influence adipose-derived stem cell function: molecular insights into impaired wound healing. Int Wound J. Mar 13, 2013 (Epub ahead of print).

46. Atiyeh BS, Amm CA and El Musa KA: Improved scar quality following primary and secondary healing of cutaneous wounds. Aesthetic Plast Surg 27: 411-417, 2003. 